\title{
EXAMEN ULTRASTRUCTURAL QUANTITATIF DES SPERMATOZOIDES HUMAINS : Intérêt, méthode, valeurs de référence, indications
}

\author{
D. Raick-Mignolet et E. Baeckeland
}

\author{
Laboratoire d'Embryologie de l'Université de Liège - 20, rue de Pitteurs, 4020 Liège (Belgique)
}

QUANTITATIVE ULTRASTRUCTURAL ANALYSIS OF HUMAN SPERMATOZOA : REVELANCE, METHOD, REFERENCE VALUES, INDICATIONS. The use of transmission electron microscopy in clinical andrology is complicated by problems of classification and interpretation related to the extraordinary pleomorphism of human spermatozoa, even in fertile men. Based upon our experience of more than 650 cases, this paper describes the morphological abnormalities seen and proposes a simplified classification which is the basis of our approach to the quantitative ultrastructural assessment of human spermatozoa. The mean and maximum incidences of the various abnormalities are provided for 37 fertile ejaculates. These reference values, particularly the threshold value of $50 \%$ abnormal acrosomes, constitute our interpretation criteria. Finally, the indications for this type of extended evaluation are defined as unexplained (idiopathic) infertility, severe asthenozoospermia, and teratozoospermia characterized by a high level of abnormal heads. Key words: Human spermatozoa, Ultrastructure, Male infertility. Andrologie 1992, 2, 114-120.

Depuis plus de trente ans, le microscope électronique à transmission a permis d'affiner considérablement la connaissance de la structure du spermatozoïde humain. L'ultrastructure du spermatozoïde normal, notamment, est très bien documentée grâce à une série de travaux remarquables $(1,23,19,8)$. Les altérations morphologiques qui peuvent affecter le spermatozoïde à l'échelle subcellulaire ont également été abondamment décrites. On trouve, dans la littérature, de très nombreuses communications rapportant des cas à vrai dire exceptionnels caractérisés par l'existence d'une anomalie ultrastructurale spécifique affectant la grande majorité voire la totalité de leurs spermatozoïdes. Il s'agit généralement de cas isolés, rarement de petites séries de cas. Citons quelques exemples de ces anomalies: spermatozoïdes à tête ronde sans acrosome (20), "miniacrosome" sperm defect: spermatozoïdes possédant un acrosome réduit (5), spermatozoïdes décapités (12), spermatozoïdes macrocéphales à flagelles multiples (17), spermatozoïdes doubles (32), "crater defect": spermatozoïdes à tête présentant une dépression apicale (4), spermatozoïdes immobiles en raison de l'absence des bras de dynéine (24), spermatozoïdes à flagelle court (9), spermatozoïdes immobiles en raison de l'absence du complexe central: " $9+0$ " (3), spermatozoïdes immobiles en raison d'une dysplasie de la gaine fibreuse (12),... La nature et le caractère généralisé de ces anomalies ont conduit à penser qu'elles étaient responsables de l'infertilité des sujets qui en étaient porteurs.

Toutefois, pour la grande majorité des sujets considérés comme infertiles, le problème de la relation entre anomalies ultrastructurales des spermatozoïdes et réduction de la fertilité s'avère beaucoup plus complexe et plus difficile à établir. On observe, en effet, chez les hommes infertiles mais également chez les sujets fertiles un certain pourcentage, plus ou moins important, de spermatozoïdes qui possèdent toute une variété de malformations associées ou non. Pour cette raison, une définition et une classification de la pathologie ultrastructurale du spermatozoïde humain en fonction, notamment, de ses conséquences fonctionnelles sont extrêmement difficiles à établir.

Ayant pressenti l'extraordinaire potentiel diagnostique et pronostique de la microscopie électronique, différents auteurs se sont néanmoins attelés à la tâche $(7,8,11,21,22,27,28$, 29) et malgré un manque d'uniformité des classifications proposées et utilisées, il ressort, de plus en plus clairement, de ces travaux qu'il existe une relation indiscutable entre la morphologie ultrastructurale des têtes spermatiques et, surtout, de l'acrosome et le pouvoir fécondant des spermatozoïdes tant in vivo qu' in vitro. L'évaluation quantitative des anomalies ultrastructurales des spermatozoïdes apparaît donc actuellement comme un outil très précieux dans l'arsenal des méthodes d'investigation de l'homme infertile. Signalons, par ailleurs, que l'utilisation des méthodes d'analyse ultrastructurale a également permis d'apprécier de manière très précise l'effet des techniques de congélation sur l'intégrité des spermatozoïdes $(16,30)$ ainsi que l'efficacité de certaines méthodes de préparation et de sélection in vitro des spermatozoïdes $(10,26)$.

Le but de ce travail est de présenter, en détails, notre propre méthode d'examen ultrastructural quantitatif ainsi que de rapporter les valeurs qui nous servent de références pour l'interprétation des résultats. Nous préciserons également les indications de cet examen.

\section{MATERIEL ET METHODES}

Les échantillons de sperme sont fournis au laboratoire, par masturbation, après une période d'abstinence de 2 ou 3 jours. Le sperme recueilli dans un récipient à usage unique est immédiatement transféré dans un tube stérile gradué, pour évaluation du volume, et laissé à température du laboratoire, à l'abri de l'air et de la lumière jusqu'à liquéfation (10 à 30 minutes). Immédiatement après ce délai, un spermogramme en microscopie photonique est réalisé et il est procédé à la fixation d'un échantillon en vue de l'étude au microscope électronique à transmission.

\section{Examen en microscopie photonique}

Pour chaque échantillon de sperme, nous estimons les paramètres suivants :

a) le volume de l'éjaculat (ml).

b) l'abondance de mucus. Nous notons 0 lorsque nous n'observons pas de mucus dans l'éjaculat. Lorsque des fils de mucus sont visibles, la note 1,2 ou 3 est donnée selon leur abondance.

c) le nombre de spermatozoïdes. Le comptage des spermatozoïdes est effectué à l'aide d'une cellule de Makler ; leur nombre, exprimé en millions par ml, est la moyenne de cinq mesures successives.

d) le pourcentage de spermatozoïdes motiles. L'évaluation du pourcentage global de spermatozoïdes motiles est réalisée dans l'heure qui suit le recueil de l'échantillon.

e) le pourcentage de spermatozoïdes morphologiquement anormaux ou tératozoospermie. La proportion de spermatozoïdes morphologiquement anormaux est évaluée sur un frottis fixé au liquide de Schaudin puis soumis successivement à la réaction de Feulgen et à une coloration au vert lumière. 200 spermatozoïdes sont examinés et classés en fonction de leurs anomalies à l'aide d'un tableau à entrées multiples selon la méthode de David et al (14).

f) le nombre de cellules rondes. Les cellules rondes, qu'elles soient des leucocytes ou des cellules germinales immatures, sont dénombrées globalement à l'aide de la cellule de Makler et le résultat est exprimé en millions $/ \mathrm{ml}$.

g) le pourcentage de spermatozoïdes morts ou nécrospermie. La proportion de spermatozoïdes morts est évaluée sur un frottis réalisé après une coloration supravitale à l'éosine-nigrosine. 
L'examen ultrastructural n'est réalisé que sur les échantillons présentant une nécrospermie inférieure ou égale à $40 \%$ ce qui fut le cas pour nos 37 témoins.

\section{Préparation des échantillons pour la microscopie électronique}

Centrifugation. En fonction de la concentration en spermatozoïdes de l'éjaculat et du volume de sperme disponible, 1 à $2 \mathrm{ml}$ de l'échantillon sont prélevés et centrifugés pendant 10 minutes à $4000 \mathrm{t} / \mathrm{min}$. En cas d'hyperviscosité, le sperme est aspiré à plusieurs reprises dans une pipette Pasteur afin de briser les fils de mucus. Après la centrifugation, le liquide séminal est éliminé et le culot cellulaire est fixé

Fixation. Le culot de spermatozoïdes est fixé dans une solution de glutaraldéhyde à $2,5 \%$ dans du tampon cacodylate $(0,1 \mathrm{M}$; pH 7,4) pendant une dizaine de minutes. Il est ensuite délicatement détaché du fond du tube à l'aide d'une fine tige de verre afin d'être fractionné en morceaux plus petits. La fixation est poursuivie pendant encore une vingtaine de minutes. Après 3 lavages dans le tampon cacodylate, on procède à la postfixation dans une solution de tétroxide d'osmium à $2 \%$ dans du tampon cacodylate, pendant 30 minutes. La fixation est réalisée à $4^{\circ} \mathrm{C}$.

Déshydratation. Les spécimens sont ensuite rincés à l'eau distillée et déshydratés dans des bains d'alcool éthylique de concentration croissante $(70 \%, 95 \%$, suivis de 3 bains à $100 \%)$ de 15 minutes chacun. Au cours des bains d'alcool $100 \%$, les échantillons sont ramenés à la température du laboratoire.

Inclusion. Après 2 bains d'époxypropane (2 fois 10 min.), les morceaux sont enchâssés dans de l'EPON.

Réalisation des coupes. Pour chaque cas, un bloc est tiré au hasard et des coupes semi-fines sont réalisées à l'aide d'un couteau de verre. Ces coupes sont examinées au microscope photonique, après coloration par le bleu de toluidine ( $1 \%$ dans l'eau), afin de vérifier la présence de spermatozoïdes en concentration suffisante. Lorsque cette condition est remplie, des coupes ultrafines $(60$ à $80 \mathrm{~nm})$ sont réalisées et recueillies sur des grilles de cuivre-rhodium (Cu/Rh 200 mailles).

Contraste des coupes. Les coupes sont contrastées à l'acétate d'uranyle (15 min.) et au citrate de plomb (15 min.).

Méthode d'examen ultrastructural quantitatif Cette méthode comporte la définition précise des critères caractérisant les anomalies que nous avons retenues et répond à la nécessité absolue d'une évaluation quantitative indépendante pour chacune de ces anomalies.

\section{L'aspect quantitatif.}

Les coupes sont examinées à l'aide d'un

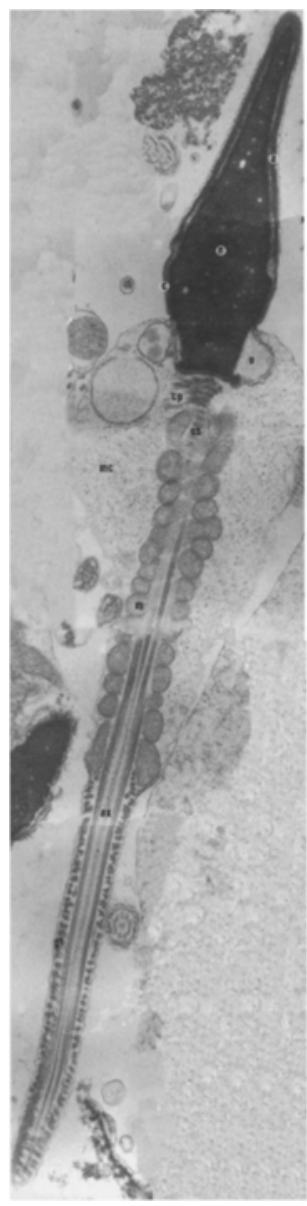

microscope électronique Philips EM 201. Les sections longitudinales de têtes sont habituellement observées à un grossissement de $6000 \mathrm{x}$ et les sections transversales de flagelles à un grossissement de $13900 \mathrm{x}$. Pour chaque cas, sur un certain nombre de plages prises au hasard, nous procédons à l'examen d'un minimum de $60 \mathrm{sec}$ tions longitudinales de tête passant par le col et d'un minimum de 60 sections transversales de flagelle: $1 / 3$ au niveau de la pièce intermédiaire et $2 / 3$ au niveau de la pièce principale. La portion tout à fait distale du flagelle (pièce terminale) n'est pas prise en considération en raison de la disparition progressive des éléments axonémaux.

Fig. 1. Section longitudinale Nos estimations des d'un spermatozoide normal. $\mathbf{n}$ taux d'anomalies sont : noyau ; a : acrosome ; $\mathbf{e}$ espace nucléaire postérieur c : cape post-acrosomique $\mathrm{CP}$ : centriole proximal ; $\mathrm{cs}$ : colonne segmentée; $\mathbf{m}$ mitochondrie ; $a x$ : axonème mc : manchon cytoplasmique ; gf : gaine fibreuse. (46 600 $x)$. Notons que la pièce principale du flagelle est, sur cette micrographie, amputée des $8 / 10$ de sa longueur réelle.

réalisées exclusivement sur base de l'examen de spermatozoïdes en bon état, c'est-à-dire, ne présentant aucun signe visible de lyse.

Nous inspirant du principe de tableau à entrées multiples proposé par David (14) pour la réalisation du spermocytogramme en microscopie photonique, nous avons établi une grille permettant de classer les têtes et les axonèmes en fonction d'une série de critères d'anomalies que nous allons détailler ci-dessous. Cette grille permet de répertorier les anomalies qu'elles soient isolées ou associées. La classification que nous utilisons regroupe les anomalies les plus fréquemment rencontrées au cours de notre pratique (plus de 650 examens réalisés à ce jour) ainsi que des anomalies beaucoup plus rares mais dont on connaît l'impact sur le pouvoir fécondant des spermatozoïdes (ex: les atteintes des bras de dynéine au niveau axonémal). Les résultats de l'examen ultrastructural quantitatif sont exprimés pour les anomalies de la tête en pourcentages de sections longitudinales de têtes en bon état et pour les anomalies de l'axonème en pourcentages de sections transversales de flagelles en bon état. Un pourcentage global des formes lysées est donné par ailleurs.

\section{$L$ 'aspect qualitatif.}

Définition des critères caractérisant les di fférentes anomalies.

\section{a. Les têtes des spermatozoïdes.}

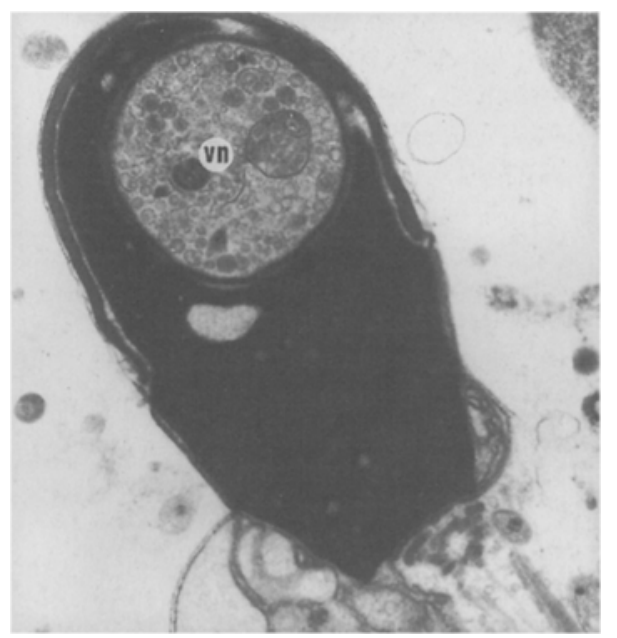

Fig. 2. Section longitudinale de tête spermatique présentant une vacuole nucléaire de grande taille, déformant le noyau et contenant du matériel granulaire (vn). (27000x).

Les têtes sont considérées comme normales et comptabilisées comme telles si elles ne présentent aucune anomalie ni signe de lyse (fig.1). Les têtes sont considérées comme lysées et comptabilisées comme telles dès que l'on y observe des signes de dégradation membranaire. Cette dégradation peut être superficielle et se limiter à la membrane plasmique ou plus profonde; des altérations des membranes acrosomiques et de l'enveloppe nucléaire sont alors visibles. Dans les cas les plus sévères, on observe une modification de la texture de la chromatine elle-même.

Nous avons retenu, pour les têtes, 6 types d'anomalies ( 3 pour le noyau, 2 pour l'acrosome et 1 pour le cytoplasme); nous allons en détailler les critères.

\section{a 1. Les anomalies du noyau :}

- Présence de vacuoles nucléaires. (Fig. 2) Nous considérons les vacuoles nucléaires comme anormales lorsqu'elles sont de taille très importante et déforment la tête du spermatozoïde ou lorsqu'elles contiennent un matériel membranaire ou acrosomique abondant. L'existence de petites lacunes "vides", n'entraînant aucune déformation du noyau est considérée comme normale.

- Trouble de la condensation chromatinienne. (Fig. 3) Nous considérons la chromatine comme mal condensée dès l'instant où elle n'apparaît pas uniformément dense aux électrons.

- Noyaux multiples. (Fig. 4) Le fait d'observer en coupe deux, trois voire quatre noyaux dans la 


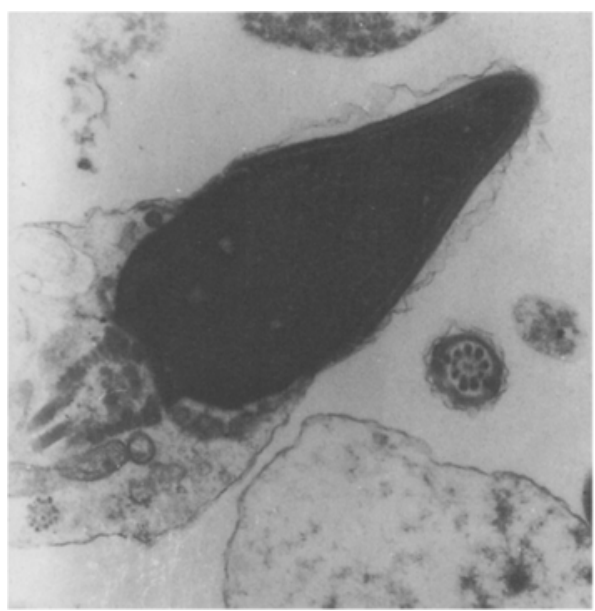

Fig. 3. Section longitudinale de tête spermatique présentant un trouble de la condensation chromatinienne. $(27.000 \mathrm{x})$.

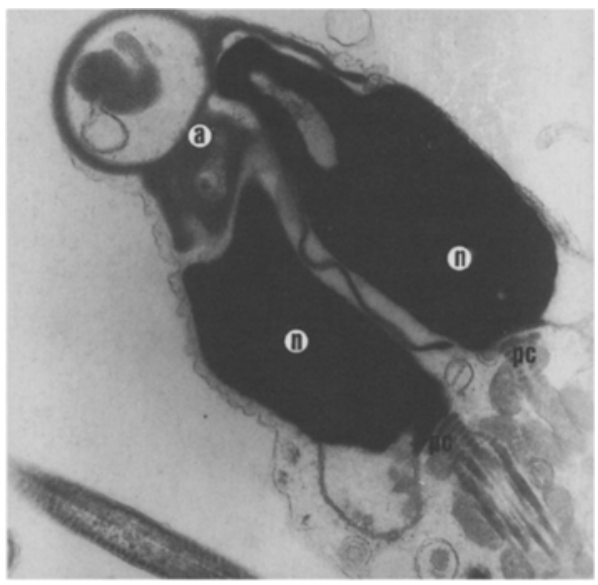

Fig. 4. Section longitudinale de tête spermatique contenant 2 noyaux (n) ; l'acrosome anormal présente diverses expansions dont une lame sinterposant entre les deux masses nucléaires (a). Chaque noyau possède sa propre pièce connective (pc). La cape post-acrosomique est absente. $(27.000 \mathrm{x})$

même tête spermatique (c'est-à-dire au sein d'un cytoplasme commun) est évidemment considéré comme anormal. Lorsque plusieurs noyaux sont présents, on observe le plus souvent l'existence d'un acrosome anormal, présentant plusieurs expansions s'insinuant entre les masses nucléaires.

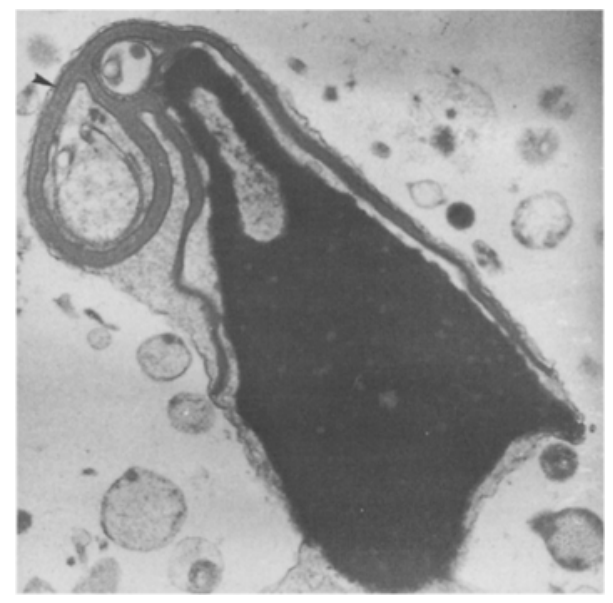

Fig. 5. Section longitudinale de tête spermatique dont l'acrosome présente une expansion apicale vacuolisée (tête de flèche). La cape post-acrosomique est absente. (27.000x).

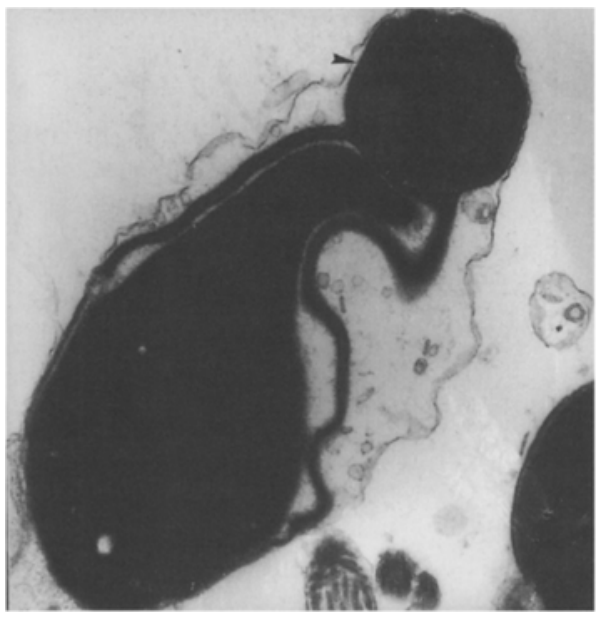

Fig. 6. Section longitudinale de tête spermatique dont l'acrosome présente une expansion apicale globuleuse (tête de flèche) $(27.000 \mathrm{x}$ ).

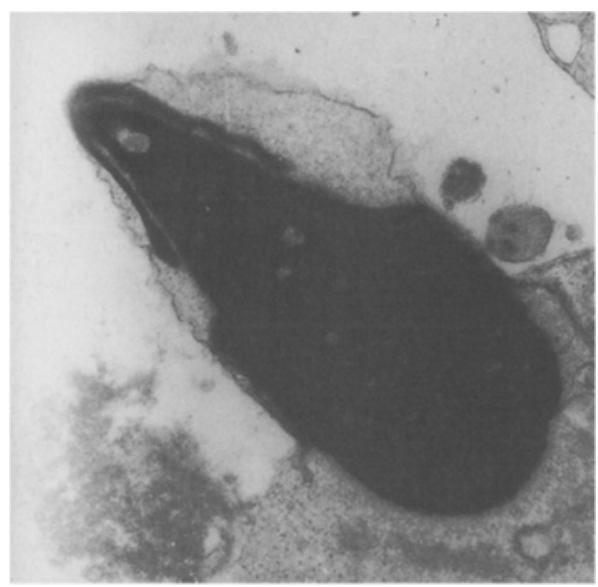

Fig. 7. Section longitudinale de tête spermatique présentant un acrosome court. Les deux segment acrosomiques ne sont pas différenciés. On note, en outre, la présence de cytoplasme résiduel et un trouble de la condensation chromatinienne. $(27000 x)$.

\section{a 2 : Les anomalies de l'acrosome :}

- Acrosome anormal.(Fig.5, 6 et 7) Il existe, en fait, une très grande variété d'anomalies de l'acrosome qui pourraient, à elles seules, faire l'objet d'une classification particulière. En pratique, nous distinguons les anomalies de structure proprement dites (expansions diverses, épaississements, raccourcissements de l'un ou l'autre segment, fragmentations,..) des anomalies de positionnement par rapport au noyau; dans ce dernier groupe,on peut observer un décollement partiel ou total de l'acrosome par rapport au noyau. Dans ce second cas, une lame de cytoplasme résiduel plus ou moins épaisse s'interpose, d'une part, entre l'acrosome et le noyau et, d'autre part, entre l'acrosome et la membrane plasmique (Fig. 12). Toutefois, dans le cadre de ce travail, nous donnons un pourcentage global reprenant toutes ces anomalies sous la rubrique: acrosome anormal.

- Réaction acrosomique précoce. Dans les spermes fraîchement éjaculés, comme ceux que nous étudions, les spermatozoïdes ayant partiellement ou totalement réalisé leur réaction acroso-

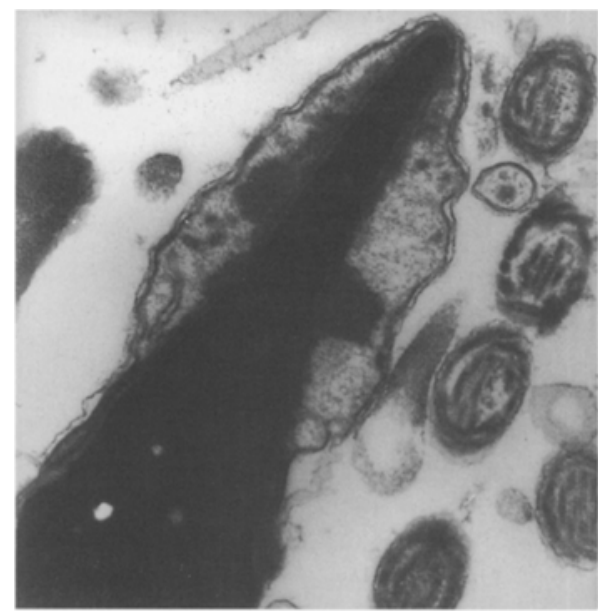

Fig. 8. Premier stade de la réaction acrosomique ; on observe un gonflement de l'acrosome ainsi qu'une décondensation partielle de son contenu. (46600x)



Fig, 9. Deuxième stade de la réaction acrosomique; on observe la formation de vésicules dans la matrice de l'acrosome à partir des membranes acrosomique inteme et externe. Notons que le segment équatorial de l'acrosome ne participe pas à la réaction acrosomique. $(46600 \mathrm{x})$

mique sont, a priori, considérés comme "anormaux". En réalité, la réaction acrosomique est, en soi, un phénomène normal et obligé du processus de fécondation ; elle n'est donc considérée, ici, comme anormale qu'en raison de son caractère précoce. Les aspects morphologiques que revêtent les spermatozoïdes en réaction acrosomique varient selon le stade atteint par le processus; ils sont rappelés dans les figures 8 à 11 .

\section{a 3 : Les anomalies du cytoplasme :}

- Présence de cytoplasme résiduel.(Fig.12) Normalement, la membrane plasmique recouvre le noyau et l'acrosome sans quasi aucune interposition de cytoplasme. Le cytoplasme existant dans la spermatide est, en effet, éliminé en grande partie au cours de la spermiogenèse ("Corps de Regaud"). La présence de cytoplasme résiduel, qu'il soit abondant ou non, qu'il englobe la tête seule ou la tête et le col, doit, par conséquent, être considérée comme anormale.

\section{b. Les flagelles des spermatozoides.}

La structure d'un flagelle normal tel qu'il apparaît en coupe transversale est rappelée dans la figure 13. 


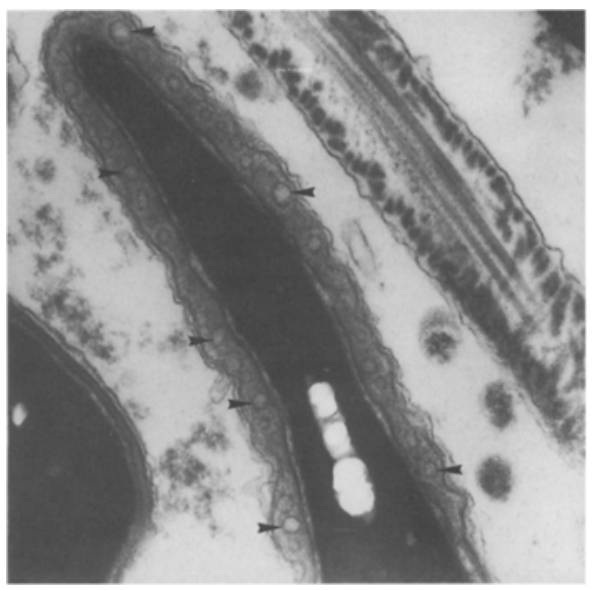

Fig. 10. Troisième stade de la réaction acrosomique ; on observe un acrosome gonflé, contenant un matếriel décondensé et de nombreuses vésicules (têtes de flèche) $(46600 \mathrm{x})$.

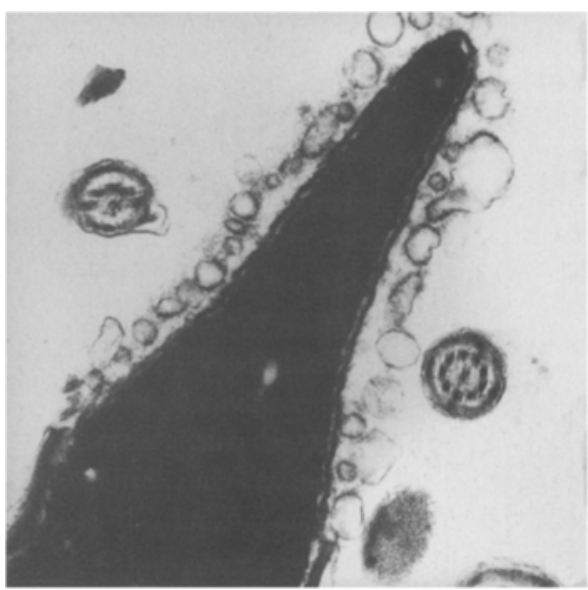

Fig. 11. Quatrième stade de la réaction acrosomique ; après fusion des membranes acrosomiques externe et plasmique, le content de l'acrosome est libéré e les véciécules se retrouvent exposées dans le voisinage immédiat du noyau sper matique. $(46600 x)$.



Fig. 12. Section longitudinale de tête spermatique possédant un cytoplasme résiduel abondant (er) qui sépare l'acrosome de la membrane plasmique. L'acrosome est partiellement décollé du noyau. La cape post-acrosomique est absente. $(27000 \mathrm{x})$

\section{b 1 : Les anomalies de l'axonème :}

Nous avons retenu, pour l'axonème, neuf types d'anomalies :

- Absence des bras internes et externes de dynéine.

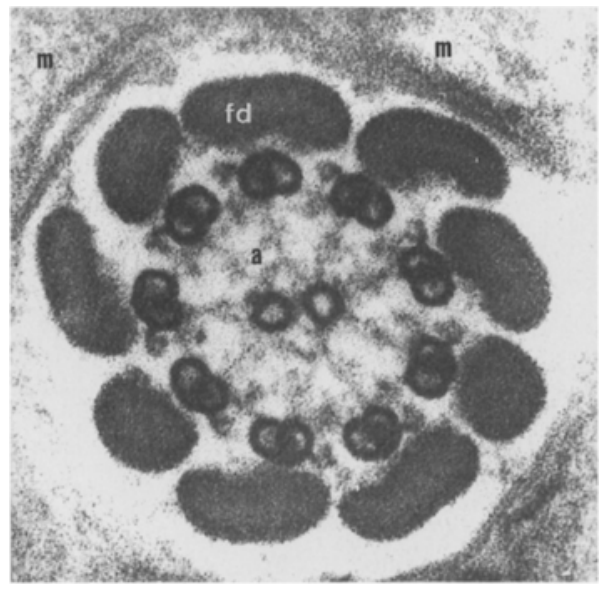

Fig. 13. Sections transversales à deux niveaux différents d'un flagelle normal. $(270000 x)$.

Photo A : Au niveau de la pièce intermédiaire, on observe autous de l'axonème (a) les $y$ fibres denses (fd) associées aux doublets et, extérieurement, le manchon de mitochondries (m)



Fig. 13. Photo B : Au niveau de la pièce principate, des fibres denses (fd) sont associées aux doublets $1,2,4,5,6,7,9$ tandis que les colonnes longitudinales (c) de la gaine fibreuse (gf) se situent en regard des doublets 3 et 8 .



Fig. 14. Section transversale de flagelle montrant une absence totale des bras externes de dynéide. (270000x).

- Absence des bras internes de dynéine et des jonctions périphériques. (liens de nexine et ponts radiaires).

- Absence des bras externes de dynéine. (fig.14)

- Absence des ponts radiaires. (fig.15). Comme ESCALIER et al.(18), nous considérons les diffé-



Fig, 15. Section transversale de flagelle montrant une absence des structures de jonction au niveau de 6 des 9 doublets (absence des bras internes et extemes de dynéine, des liens de nexine et des ponts radiaires). (270000x).

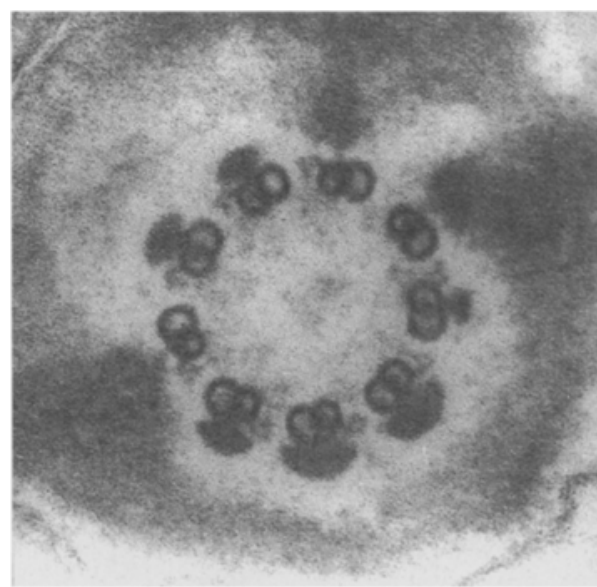

Fig. 16. Section transversale de flagelle dont l'axonème montre une absence du complexe central. $(270000 \mathrm{x})$

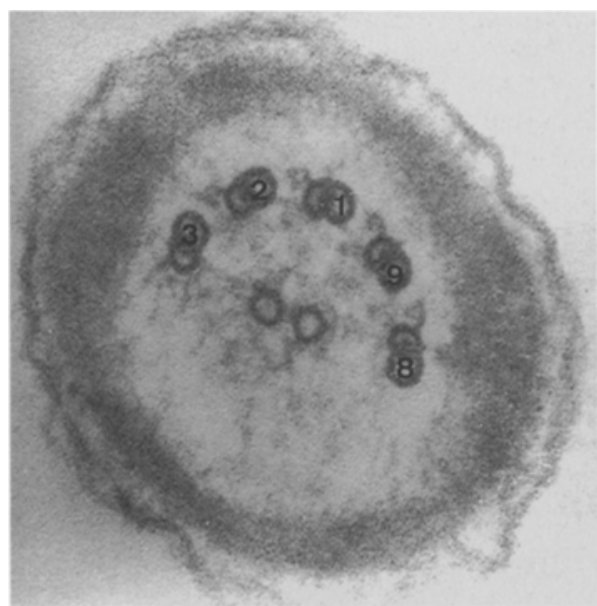

Fig. 17. Section transversale de flagelle dont l'axonème montre l'absence des doublets $n^{\circ} 4,5,6$ et 7 . (270000x).

rents éléments ci-dessus comme absents d'une section, s'ils manquent au moins au niveau de 6 des 9 doublets.

- Absence du complexe central. (fig.16). Le complexe central est considéré comme absent dès que l'un de ses éléments est absent (gaine centrale, un des tubules). Ces cas sont toutefois exceptionnels; on note le plus souvent une absence de 


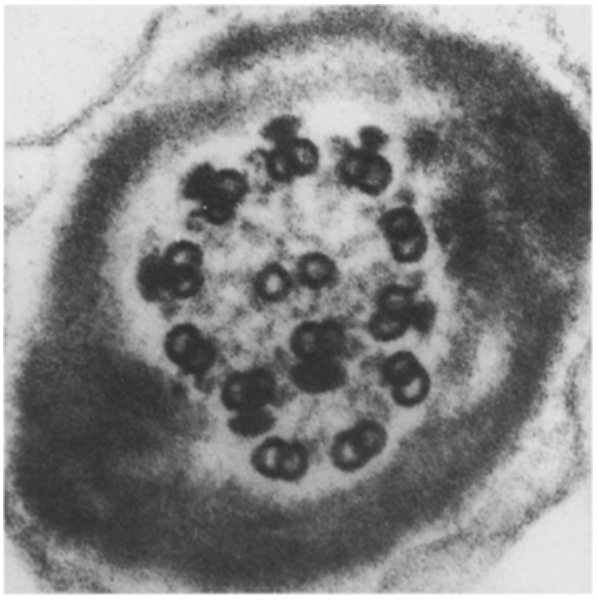

Fig. 18. Section transversale de flagelle montrant la présence de trois doublets surnuméraies au niveau de la pièce principale. (270000x).



Fig. 19. Section transversale de flagelle montrant une désorganisation totale des éléments axonémaux. 5 doublets seulement sont présents dont 3 en position anomale, en dehors de la gaine fibreuse. (270000x).

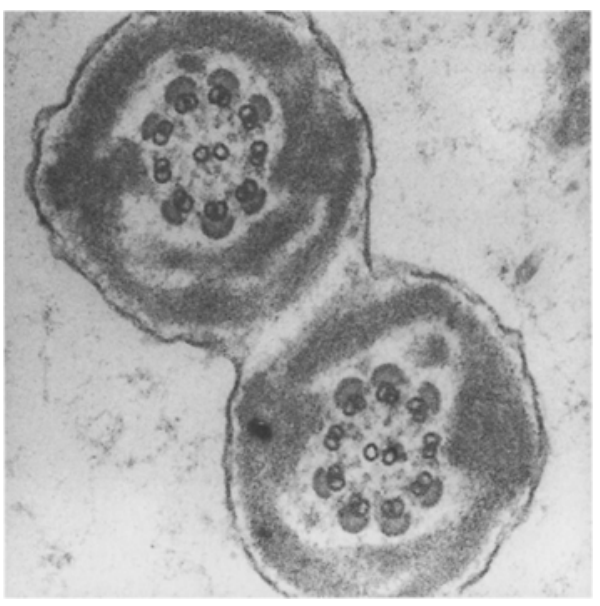

Fig. 20. Section transversale de flagelle possédant deux axonèmes normaux (110000x).

l'ensemble de la structure.

- Absence d'un ou de plusieurs doublets et des fonctions périphériques correspondantes. (fig.17). Dès l'instant où un doublet est absent, l'axonème est considéré comme anormal. Nous notons le nombre de doublets manquants.

- Présence de doublets surnuméraires. (fig.18)

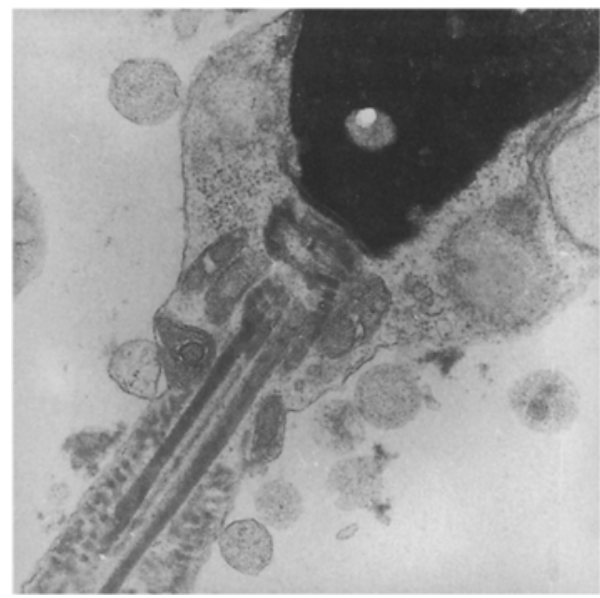

Fig. 21. Section longitudinale de flagelle possédant une gaine mitochondriale courte et désorganisée. (46600x).

Nous notons le nombre de doublets surnuméraires qu'ils soient localisés au sein de l'axonème proprement dit ou en dehors de l'appareil périaxonémal.

- Désorganisation totale. (fig 19) Nous considérons l'axonème comme totalement désorganisé lorsque ses éléments constitutifs sont déplacés les uns par rapport aux autres de telle manière que la disposition circulaire normale des doublets est perturbée. Cette anomalie résulte de l'absence de structures de jonction et s'accompagne le plus souvent d'altérations du nombre de doublets.

- Axonèmes multiples. (fig.20) Nous comptabilisons, sous ce vocable, toutes les sections de flagelles contenant au moins deux axonèmes normaux ou non.

\section{b 2 : Les anomalies du périaxonème :}

- Désorganisation de la gaine mitochondriale. (fig.21) Celle-ci est considérée comme désorganisée lorsque les mitochondries sont disposées en plusieurs couches ou dispersées dans un cytoplasme résiduel abondant localisé au niveau de la pièce intermédiaire.

- Absence d'une ou des deux colonnes longitudinales au niveau de la gaine fibreuse. Elle est considérée comme anormale si elle se manifeste

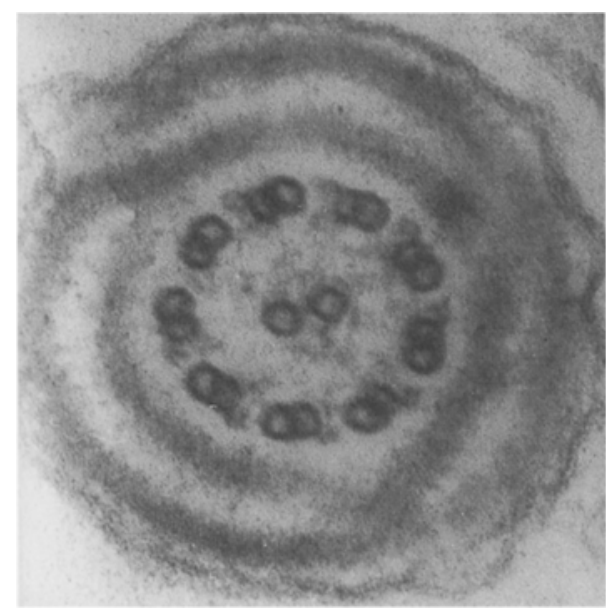

Fig. 22. Section transversale de flagelle possédant une gaine mitochondriale courte et désorganisée. $(270.000 \mathrm{x})$. soit au niveau de la pièce intermédiaire soit au niveau de la partie proximale de la pièce principale.

- Epaississement de la gaine fibreuse. (fig.22) Nous désignons sous cette appellation les gaines fibreuses constituées de plus d'une couche d'arceaux semi-circulaires.

Nos valeurs de référence - c'est-à-dire les valeurs dont nous nous servons pour interpréter les résultats des examens ultrastructuraux des patients qui nous sont adressés - ont été établies à partir des résultats des examens de sperme de 37 sujets fertiles. Il s'agit, d'une part, de 19 sujets âgés de 23 à 35 ans dont la fertilité était attestée par une conception récente non médicalement assistée (témoins fertiles "pères") ; 12 d'entre eux étaient pères d'un enfant âgé de moins de 1 an au moment de l'examen et 7 étaient pères de 2 enfants dont le plus jeune était âgé de moins de 18 mois - et, d'autre part, de 18 sujets âgés de 25 à 50 ans dont la fertilité était attestée par un taux de fécondation élevé (plus de $2 / 3$ des ovocytes fécondés) lors d'une tentative de F.I.V.(témoins fertiles "FIV"). Ces sujets étaient engagés dans un programme F.I.V. en raison d'une indication exclusivement féminine (problème tubaire : 14 cas, problème endocrinien: 3 cas, endométriose: 1 cas).

\section{RESULTATS}

Les résultats des deux séries de témoins fertiles: témoins fertiles "pères" et témoins fertiles "FIV" ont été comparés. Les valeurs observées dans les deux séries étaient très proches et une analyse statistique consistant, d'une part, en un test $t$ de Student et, d'autre part, en une analyse multidimensionnelle discriminante logistique (2) a montré que les sujets de ces deux séries appartenaient à une même population et pouvaient être regroupés en un seul groupe de témoins fertiles dont les paramètres spermatiques moyens en microscopie photonique sont donnés dans le tableau I. Les valeurs de référence que nous utilisons pour la microscopie électronique sont les moyennes et surtout les valeurs maximales obtenues pour les différents paramètres ultrastructuraux sur ces 37 spermes fertiles; elles sont présentées dans le tableau II.

Tableau I - Paramètres spermatiques des témoins fertiles

\begin{tabular}{|l|c|}
\hline Nombre de sujets & 37 \\
Age moyen & $31,4(6,2)$ \\
Volume du sperme (ml) & $3,3(1,4)$ \\
Présence de mucus & $0,6(0,9)$ \\
Nombre de spermatozoïdes $(\mathrm{M} / \mathrm{ml})$ & $124,2(58,6)$ \\
Motilité $(\%)$ & $73,2(13,2)$ \\
Tératozoospermie $(\%)$ & $35,6(12,4)$ \\
Nombre de cellules rondes $(\mathrm{M} / \mathrm{mi})$ & $2,2(3,8)$ \\
Nécrospermie $(\%)$ & $15,1(8,8)$ \\
\hline
\end{tabular}

Les valeurs sont les moyennes (écarts-types)

Le pourcentage moyen de formes lysées chez les témoins fertiles est de 13,2+11,4\% (valeur 
Tableau II - Valeurs de référence : Paramètres ultrastructuraux des témoins fertiles

\begin{tabular}{|c|c|c|c|}
\hline & Moyennes (SD) & Minima & Maxima \\
\hline \% global de spermatozoïdes lysés & $13,2(11,4)$ & 0 & 47 \\
\hline \% global de têtes anormales & $46,0(12,4)$ & 25 & 75 \\
\hline \% global d'axonèmes anormaux & $31,5(14,1)$ & 8 & 78 \\
\hline \% global d'anomalies periaxonémales & $0,2(1,0)$ & 0 & 6 \\
\hline \multicolumn{4}{|l|}{ DETAIL DES ANOMALIES DE LA TETE $(\%)$} \\
\hline - présence de vacuoles nucléaires & $6,6(5,1)$ & 1 & 20 \\
\hline - trouble de la condensation chromatinienne & $12,0(11,7)$ & 0 & 58 \\
\hline - noyaux multiples & $3,1(3,5)$ & 0 & 15 \\
\hline - acrosomes anormaux & $20,6(8,6)$ & 8 & 48 \\
\hline - réactions acrosomiques précoces & $7,6(9,6)$ & 0 & 48 \\
\hline - présence de cytoplasme résiduel & $16,9(7,4)$ & 6 & 36 \\
\hline \multicolumn{4}{|l|}{ DETAIL DES ANOMALIES DE L'AXONEME (\%) } \\
\hline - abs. des bras internes et externes de dynéine & $0,05(0,3)$ & 0 & 2 \\
\hline - abs. des bras internes de dynéine et des jonctions périphériques & 0 & 0 & 0 \\
\hline - absence des bras externes de dynéine & $0,5(2,8)$ & 0 & 17 \\
\hline - absence des ponts radiaires & $0,7(1,2)$ & 0 & 4 \\
\hline - absence du complexe central & $4,3(4,8)$ & 0 & 17 \\
\hline - absence de doublets & $20,5(11,3)$ & 2 & 49 \\
\hline - présence de doublets sumuméraires & $2,0(1,9)$ & 0 & 7 \\
\hline - désorganisation totale & $4,3(3,9)$ & 0 & 13 \\
\hline - axonèmes multiples & $1,1(1,7)$ & 0 & 6 \\
\hline
\end{tabular}

maximale $47 \%$ ). Les pourcentages globaux d'anomalies de la têtes et de l'axonème s'élèvent respectivement à $46,0+12,4 \%$ (valeur maximale $75 \%$ ) et $31,5+14,1 \%$ (valeur maximale $78 \%$ ). Les anomalies les plus fréquemment observées au niveau de la tête des spermatozoïdes sont : 1) les anomalies de l'acrosome $20,6+8,6 \%$ (valeur maximale $48 \%$ ), 2) la présence de cytoplasme résiduel $16,9+7,4 \%$ (valeur maximale $36 \%$ ), 3 ) les troubles de la condensation chromatinienne $12,0+11,7$ (valeur maximale $58 \%$ ). Au niveau des axonèmes, l'anomalie la plus fréquemment observée est l'absence d'un ou de plusieurs doublets et leurs jonctions $20,5+11,3 \%$ (valeur maximale $49 \%$ ). Toutes les autres anomalies sont extrêmement rares chez les témoins fertiles puisque nous les observons avec une fréquence moyenne inférieure à $5 \%$. Notons, tout particulièrement, la quasi absence d'anomalies touchant les bras de dynéine.

\section{DISCUSSION}

L'utilisation de la microscopie électronique pour l'évaluation du pouvoir fécondant du sperme est trop récente et limitée à trop peu de laboratoires pour que l'on puisse espérer, dès à présent, une uniformisation des méthodes de travail et des critères d'interprétation. Tout comme pour la microscopie photonique, cette uniformisation demandra vraisemblablement beaucoup de temps et de discussions. C'est la raison pour laquelle les valeurs de référence que nous rapportons dans ce travail sont difficilement comparables à celles des autres auteurs. En ce qui concerne par exemple le pourcentage global d'anomalies de la tête chez les sujets fertiles, nos résultats sont nettement moins élevés que ceux obtenus par Escalier et al.(16) ou Navarro et al.(22). Ceci est essentiellement dû au fait que ces auteurs reprennent parmi les anomalies de la tête les anomalies de la forme générale du noyau même conclusion mais nous avons, en outre, pu préciser qu'au-dessus d'un seuil de $50 \%$ d'acrosomes anormaux les chances d'obtenir une fécondation seraient extrêmement réduites. Endessous de ce seuil, la motilité des spermatozoïdes et le pourcentage de têtes spermatiques présentant des troubles de la condensation chromatinienne paraissent jouer un rôle déterminant. La valeur prédictive de ce seuil paraît validée par le fait que nous ayons observé une fréquence maximale de $48 \%$ d'acrosomes anormaux dans notre série de témoins fertiles. L'impact sur le pouvoir fécondant et la valeur prédictive d'autres paramètres tels que le pourcentage d'anomalies de la cape post-acrosomique sont actuellement en cours dans notre laboratoire.

D’ores et déjà, grâce aux résultats récents rapportés cidessus et aux critères d'interprétation qui commencent à émerger de ces travaux, le microscope électronique devient, de plus en plus, un outil capable de rendre des services appréciables aux praticiens. Néanmoins l'examen ultrastructural quantitatif est un examen spécialisé dont l'interprétation reste souvent difficile. Il s'agit, en outre, d'une technologie relativement lourde tant du point de vue du coût que de celui du temps de mise en oeuvre. Pour ces raisons, il convient de le réserver à des indications très précises.

\section{INDICATIONS DE L'EXAMEN ULTRASTRUCTURAL QUANTITATIF DU SPERME.}

Selon nous, ce type d'examen est indiqué dans les cas d'infertilité de longue durée ou dans les cas d'échecs de fécondation in vitro mais, uniquement, lorsque le diagnostic d'infertilité masculine n'a pu être établi, de manière patente, par le spermogramme classique en microscopie photonique. Dans ces cas, seul l'examen ultrastructural permettra de mettre en évidence ou d'exclure l'existence d'anomalies morphologiques qui ne sont pas détectables lors du spermocytogramme en microscopie photonique mais dont l'impact sur le pouvoir fécondant est actuellement bien établi. Nous pensons que l'examen ultrastructural du sperme pourrait toutefois être réalisé de manière systématique lorsque la fécondation in vitro est envisagée pour pallier à une subfertilité masculine reconnue. Cohen et al.(13) ont, certes, montré que de nombreux hommes ayant un sperme anormal étaient capables de féconder des ovocytes humains in vitro; il n'en reste pas moins que l'on observe en cas de fécondation in vitro avec des oligo-asthéno-tératozoospermes, un taux d'échecs complets de fécondation pouvant atteindre de 26 à $45 \%$ $(23,6)$. L'échec de fécondation étant certainement l'événement le plus frustrant qui puisse survenir tant pour le couple que pour l'équipe soignante, il ne nous paraît pas superflu de tenter de le pré- 
venir en réalisant préalablement une évaluation la plus précise possible du pouvoir fécondant du sperme notamment dans les cas de tératozoospermie élevée au niveau des têtes spermatiques. La microscopie électronique va permettre de dénombrer très précisément la proportion de têtes normalement constituées et d'évaluer l'état des acrosomes afin de juger s'il est légitime d'orienter le couple vers telle ou telle technique de procréation médicalement assistée. Il va sans dire que, dans tous les cas, la part de responsabilité féminine dans le problème d'infertilité doit, bien entendu, être rigoureusement évaluée et prise en compte. Enfin, l'examen ultrastructural des axonèmes a une valeur diagnostique incontestable dans les cas d'asthénozoospermie sévére.

\section{REFERENCES}

1 - Afzelius B. Electron microscopy of the spermtail. Results obtained with a new fixative. J. Biophysic. Biochem. Cytol., 1959,5:269-278.

2 - Albert A, Lesaffre E. Multiple group logistic discrimination. Comp. Maths with Appls, 1986, 12A:209-224.

3 - Baccetti B., Burrini AG., Maver A., Pallini V., Renieri T. "9+0" Immotile spermatozoa in an infertile man. Andrologia, 1979, 11:437-443.

4 - Baccetti B., Burrini AG., Collodel G., Magnono AR., Piomboni P., Renieri T., Sensini C. Crater defect in human spermatozoa. Gamete Res., 1989, 22:249-255.

5 - Baccetti B., Burrini A.G., Collodel G., Piomboni P., Renieri T. A "Miniacrosome" sperm defect causing infertility in two brothers. J. Androl., 1991, 12:104-111.

6 - Barlow P., Englert Y., Puissant F., Lejeune B., Delvigne A., Van Rysselberge M., Leroy F. Fertilization failure in IVF: why and what next? Human Reprod., 1990, 5:451-456.

7 - Bartoov B., Eltes F., Weissenberg R., Lunenfeld A. Morphological characterization of abnormal human spermatozoa using TEM. Arch. Androl., 1980, 5:305-322.

8 - Bisson JP., David G. Anomalies morphologiques du spermatozoïde humain. 2) Etude ultrastructurale. J. Gyn. Obst. Biol. Repr.,Suppl., 1975, 1:37-86.

9 - Bisson JP., Leonard C., David G. Caractère familial de certaines perturbations morphologiques des spermatozoïdes. Arch. Anat. Cytol. Path., 1979, 27:230-233.

10 - Bower L., Serafini P., Wikland M., Moyer D. The use of hyaluronic acid for sperm selection Evaluation of sperm head ultrastructure. Int. J. Fertil., 1989, 34:420-424.

11 - Carlon N., Navarro A.,Pizant J. Etude ultrastruturale quantifiée du sperme dans 72 cas d'infcondité masculine. Contracept. Fertil. Sex., 1991, 19:985-993.
12 - Chemes HE., Carizza C., Scarinci F., Brugo S., Neuspiller N., Schwarstein T. Lack of a head human spermatozoa from sterile patients: a syndrome associated with impaired fertilization. Fertil. Steril., 1987, 47:310-316.

13 - Cohen J., Edwards R., Fehilly C., Eishel S., Hewitt J., Purdy J., Rowland G., Steptoe P., Webster J. In vitro fertilization: a treatment for male infertility. Fertil. Steril., 1985, 43:422-432.

14 - David G., Bisson JP., Czyglik F., Jouannet P., Gernigon C. Anomalies morphologiques du spermatozoïde humain. 1) Proposition pour un système de classification. J. Gyn. Obst. Biol. Repr. Suppl., 1975, 4:17-36.

15 - David G., Escalier D. Apport de la microscopie électronique à l'examen du sperme. In: A la recherche du pouvoir fécondant du sperme. Schoysman R (eds), 2ème Congrès de la S.A.L.F, Bruxelles, 1897, 57-65.

16 - Escalier D., Bisson JP. Quantitative ultrastructural modifications in human spermatozoa after freezing. In: Human artificial insemination and semen preservation. David G.and Price WS. (eds), Plenum Press (New York), 1980, 107-122.

17 - Escalier D. Human spermatozoa with large heads and multiple flagella: quantitative ultrastructural study of 6 cases. Biol. Cell., 1983, 48:65-74.

18 - Escalier D., David G. Pathology of the cytoskeleton of the human sperm flagellum : Axonemal and periaxonemal anomalies. Biol. Cell., 1984, 50:37-52.

19 - Fawcett DW. Review Article. The mammalian spermatozoon. Dev. Biol., 1975, 44:394-436.

20 - Jeyendran RS., Van Der Ven HH., Kennedy WP., Perez-Pelaes M., Sobrero AJ., Zaneveld LJD. Acrosomeless sperm. A cause of primary male infertility. Andrologia, 1985, 17:31-36.

21 - Mashiach R., Fisch B., Eltes F., Tadir Y., Ovadia J., Bartoov B. The relationship between sperm ultrastructural features and fertilizing capacity in vitro. Fertil. Steril., 1992, 57:1052-1057.

22 - Navarro A., Pizant J., Carlon N. Ultrastructure of spermatozoa in unexplained failure of IVF. Mol. Androl., 1992, 4:72-80.

23 - Pedersen $H$. Ultrastructure of the ejaculated human sperm. Z. Zellforsch., 1969, 94:542-554.

24 - Pedersen H., Rebbe H. Absence of arms in the axoneme of immobile human spermatozoa. Biol. Reprod., 1975, 12:541-544.

25 - Plachot M., Mandelbaum J., Junca AM. Moyens actuels d'évaluation de la fécondance du sperme. Contracep. Fertil. Sexualité, 1986, 14:211-219.

26 - Raick D. Détermination et évaluation quantitative de critères ultrastructuraux permettant une esti mation du pouvoir fécondant des spermatozoïdes humains. Doctorat en Sciences Biomédicales Expérimentales, Université de Liège, 1991, 207
27 - Raick D., Baeckeland E. Letawe J. A quantitative study of ultrastructural spermatozoa defects in relation with infertility. Prog. Reprod. Biol. Med. Basel, Karger, 1992, 15 194-201.

28 - Raick D., Baeckeland E. Predictive value of electron microscopy of sperm for IVF. Mol. Androl., 1992, 4:72-80.

29 - Renieri T. Submicroscopal observations on abnormal human spermatozoa. J. Submicr. Cytol., 1974, 6:421-432.

30 - Serafini P., Hauser D., Moyer D., Marrs RP. Cryopreservation of human spermatozoa: correlations of ultrastructural sperm head configuration with sperm motility and ability to penetrate zona free hamster ova. Fertil. Steril., 1986, $46: 691$ 695 .

31 - Zamboni L. The ultrastructural pathology of the the spermatozoon as a cause of infertility: the role of electron microscopy in the evaluation of semen quality. Fertil. Steril,, 1987, 48:711-734.

32 - Zukerman Z., Sagiv M., Ravid A., Ben-Bassat M., Malik Z., Shohat B., Tadir Y., Ovadia Y., Singer R. A high proportion of double-headed and double-tailed sperm in semen of a human male. A case report. Andrologia, 1986, 18: 495-501.

RESUME : L'utilisation de la microscopie électronique à transmission en clinique andrologique se heurte à des difficultés de classification et d'interprétation liées à l'extraordinaire pléiomorphisme caractérisant les spermatozoïdes humains et ce, même chez les sujets fertiles. Forts d'une expérience portant sur plus de 650 cas, nous donnons ici une description des malformations observées et proposons la classification simplifiée servant de base à notre méthodologie d'examen ultrastructural quantitatif du sperme humain. Nous rapportons les fréquences moyennes et maximales de ces malformations dans 37 spermes fertiles. Ces valeurs de référence et notamment la valeur seuil de $50 \%$ d'acrosomes anormaux constituent nos critères d'interprétation. Nous précisons, enfin, les indications de ce type d'exploration approfondie à savoir: les infertilités inexpliquées, les asthénozoospermies sévères et les tératozoospermies caractérisées par un taux élevé de têtes anormales. Mots clés : Spermatozoïde humain, Ultrastructure, Infertilité masculine. Andrologie, 1992, 2 : 114-120. 